\title{
Philosophiques
}

\section{Commentaire sur Décoloniser le féminisme de Soumaya Mestiri}

\section{DIANE LAMOUREUX}

Volume 44, numéro 1, printemps 2017

URI : https://id.erudit.org/iderudit/1040332ar

DOI : https://doi.org/10.7202/1040332ar

Aller au sommaire du numéro

Éditeur(s)

Société de philosophie du Québec

ISSN

0316-2923 (imprimé)

1492-1391 (numérique)

Découvrir la revue

Citer ce document

Lamoureux, D. (2017). Commentaire sur Décoloniser le féminisme de Soumaya

Mestiri. Philosophiques, 44(1), 117-121. https://doi.org/10.7202/1040332ar d'utilisation que vous pouvez consulter en ligne.

https://apropos.erudit.org/fr/usagers/politique-dutilisation/ 


\title{
Commentaire sur Décoloniser le féminisme de Soumaya Mestiri
}

\author{
DIANE LAMOUREUX \\ Département de science politique \\ Université Laval \\ Diane.Lamoureux@pol.ulaval.ca
}

Dans cet ouvrage, Soumaya Mestiri propose de décoloniser le féminisme, en adoptant la perspective d'un féminisme de la frontière s'inspirant des femmes chicanas et des théories de la colonialité du pouvoir, et en ancrant ses réflexions dans les réalités tunisiennes de l'après 20II. Le projet est ambitieux et, dans cet ouvrage, somme toute assez court, elle nous livre une esquisse d'un programme intellectuel de longue haleine, abordant des enjeux assez diversifiés. Pour les fins du présent exercice, je me prêterai à une réflexion en trois temps: d'abord, je discuterai de la perspective générale qui nous est proposée tant dans le prologue que dans l'épilogue; ensuite, je me pencherai sur la critique qu'elle propose de l'empowerment (chapitre V); finalement, je discuterai du care (chapitre VI) comme réponse aux limites de l'empowerment.

Il me semble utile de préciser le point de vue d'où je parle. Mon entrée dans le féminisme s'est faite sur le plan militant, dans les années I970, à Montréal. Ensuite, j'ai fait une carrière universitaire de professeure au Département de science politique de l'Université Laval, principalement dans le domaine de la philosophie politique, tout en gardant le féminisme comme point d'ancrage. Mes travaux portent principalement sur le féminisme québécois, mais également sur les théories féministes. Au cours des dernières années, je me suis intéressée plus sérieusement au féminisme noir étasunien et aux théories décoloniales, tout en n'étant pas une spécialiste de ces domaines.

\section{Un féminisme de la frontière}

L'idée d'un féminisme de la frontière a beaucoup été portée par des féministes chicanas qui avaient l'impression d'être situées entre deux mondes et qui ne voulaient renoncer à aucun de ces deux mondes, la renonciation impliquant une amputation d'une part d'elles-mêmes. Un tel féminisme constitue une remise en cause salutaire d'une partie de la tradition féministe occidentale qui, principalement dans sa variante libérale, postulait un universalisme qui reconduisait l'hégémonie occidentale sur la planète. Rompre avec ce faux universalisme oblige, comme nous y convie justement Mestiri, à repenser les conditions de la solidarité féministe afin que le projet féministe contribue à combattre l'ensemble des dominations. 
Contrairement à un imaginaire dominant de la frontière qui en fait une clôture, Mestiri privilégie, à la suite de Lugones mais aussi de penseurs décoloniaux comme Mignolo, ou occidentaux comme Balibar, de penser la frontière comme un point de contact, une position instable, transversale et poreuse. Ainsi, l'auteure privilégie la borne plutôt que la limite.

Dans cette perspective, il est possible de conceptualiser de manière nouvelle les rapports entre la marge et le centre. À partir du moment où l'on perçoit le centre comme le résultat d'une position hégémonique, la solution n'est évidemment pas de faire de la marge un nouveau centre puisque, ce faisant, nous ne ferions que déplacer les termes de la domination sans toucher à la domination elle-même. Il s'agit plutôt de procéder à un décentrement général, de faire en sorte que le centre puisse rester vide afin de mettre en évidence et d'entreprendre de corriger les injustices épistémiques et sociales.

Cela permet également de concevoir les marges dans leur diversité. Reprenant à son compte l'analogie de Dussel entre le « je pense donc je suis » et «je conquiers donc je suis", Mestiri soutient que ce "sont les deux faces d'une même stratégie épistémique qui dissimule et soustrait le sujet particulier, local, indigène, au profit du sujet universel, blanc, impérialiste de l'énonciation » (p. I6). Ce qui pose problème dans l'universel, c'est justement cette volonté de ramener à du "un ", ce qui se présente toujours spontanément sous la forme du multiple.

Pour déstabiliser le «un» hégémonique, Mestiri nous propose de prendre au sérieux les savoirs produits par les subalternes et de faire en sorte qu'ils puissent dialoguer à égalité avec les savoirs hégémoniques afin de mettre en lumière le caractère partiel et partial de ces derniers. Ce dialogue à égalité suppose une rupture qui permette la transversalité des échanges (p. I6). Il impose également de prendre en considération des savoirs situés, "une re-connection de l'épistémologie et de la production du savoir avec la localisation ethnique, sexuelle et raciale de celles et ceux qui pensent et qui parlent» (p. I7).

Ainsi, il serait possible de remplacer l'hégémonie universaliste par ce que je qualifierais de pluriversalisme, un travail constant de traduction entre l'incommensurabilité des expériences et la nécessité de construire des coalitions qui permettent de décentrer le centre. Mestiri parle, pour sa part, reprenant encore là Lugones, de «multiculturalisme radical» ou de «multiculturalisme pluricentré» (p. I 58 ), et soulève la possibilité, sur cette base, d'une histoire plurielle mais partagée qui préserve la dignité de toutes et tous.

Le travail de solidarité et le féminisme inclusif que Mestiri appelle de ses vœux est, comme nous le rappellent plusieurs féministes noires étasuniennes, un processus sans fin, un dialogue infini, un "féminisme sans frontières qui croise sans réifier et qui comprend sans réduire»(p. I9). Nous sommes assez loin à la fois de la tolérance libérale qui hiérarchise, et de l'hybridation postmoderne qui délocalise. C'est ce qui me rend perplexe face 
à la présence dans l'ouvrage d'un essai critique sur une partie du travail de Rawls et d'un autre sur John Stuart Mill, ce qui s'explique probablement par la tension, soulignée par l'auteure "entre la volonté, bien réelle, de sortir de la problématique libérale et la difficulté qu'il y a à ne pas y demeurer » (p. I 57). Pour moi, qui n'appartiens pas à cette tradition libérale, c'est un point de vue assez difficile à partager, d'autant plus que le libéralisme est, dans le monde contemporain, le principal porteur du «un» hégémonique critiqué dans cet ouvrage.

\section{Quel empowerment?}

Je partage entièrement la position de Mestiri qui vise à décoloniser l'empowerment parce que, dans la version libérale endossée par la Banque mondiale ou les ONG internationales, il ne fait que reconduire la sujétion des groupes subalternes. Toutefois, il me semble que l'une des façons de procéder est de remonter à l'origine de la notion et à en dénier la paternité (ou maternité) aux institutions internationales qui l'ont captée et détournée à leur profit dans leurs fumeux «objectifs du millénaire».

Car la notion d'empowerment a une longue histoire qui remonte à la fois aux luttes des femmes et aux luttes des Noir.es aux États-Unis. Comme le rappelle, notamment, la féministe noire étasunienne Patricia Hill Collins, promouvoir l'empowerment des Africaines-Américaines implique de travailler simultanément sur les dimensions institutionnelle, disciplinaire, hégémonique et interpersonnelle du pouvoir, et d'inscrire le projet dans une perspective plus générale de justice sociale transnationale. Cela rend nécessaire de prendre en considération la multiplicité des efforts déployés par les personnes appartenant à divers groupes sociaux minorisés pour arriver à se définir soi-même plutôt que d'être défini.e par les groupes dominants, de faire confiance à sa propre perception de son expérience et d'en faire une source de savoir sur les réalités sociales, d'utiliser toutes les failles du système, à quelque niveau qu'elles se situent, pour résister à la dévalorisation et affirmer sa dignité sur le plan à la fois personnel et collectif, et de mener des luttes collectives pour changer les rapports de force. À l'origine, articulée à des projets d'émancipation sociale, la notion d'empowerment était éminemment politique, et sa captation par diverses organisations internationales dans le cadre de la lutte à la pauvreté a consisté en un double mouvement de dé-radicalisation et de dé-politisation. Décoloniser la notion implique donc de rappeler et valoriser ses origines et non se contenter de l'ersatz d'empowerment que critique fort justement Mestiri.

Celle-ci nous montre le lien entre le discours néolibéral sur l'empowerment et les diverses stratégies de lutte à la pauvreté. Il me semble qu'il faut également critiquer ces stratégies qui visent moins à sortir les femmes de la pauvreté qu'à les inscrire dans le cycle de l'économie formelle néolibérale et ainsi contribuer à sa reproduction élargie à l'échelle internationale. À cet égard, la critique développée par Sen en ce qui concerne la dissociation entre 
la satisfaction des besoins matériels et les libertés politiques est tout à fait justifiée, même si elle est insuffisante. Certes, les droits humains sont insécables et acquièrent une certaine efficacité politique si on considère comme un ensemble les droits civils, politiques, économiques, sociaux et culturels. Mais la structure même de la propriété et du pouvoir politique dans les sociétés capitalistes à l'ère néolibérale nous oblige à considérer la pauvreté non pas comme une situation mais comme un rapport social inégalitaire.

À cet égard, je ne peux que partager la critique de Mestiri par rapport à une vision en cinq étapes de l'empowerment (p. I Io) et à l'utilisation de la notion dans les politiques de coopération internationale les mieux intentionnées qui adoptent la perspective "genre et développement", politiques qui échouent à dépasser la société civile pour s'attaquer aux structures du pouvoir et de la domination, et à sa limitation à former certaines femmes à s'insérer dans l'élite du pouvoir sans remettre en cause le caractère élitiste du pouvoir, comme il est bien montré aux chapitres III et IV de l'ouvrage en utilisant l'exemple tunisien.

Toutefois, je ne suis pas particulièrement convaincue par les arguments qu'elle avance dans la troisième partie du chapitre $V$, même si je partage le projet qu'elle met de l'avant, "émanciper les femmes de toute forme de tutelle " (p. I I9), ce qui implique fort justement de "transformer la société » (p. I I9). Si j'adhère sans réserve à l'idée de rompre avec les politiques officielles de lutte à la pauvreté en raison du caractère insécable de l'ensemble des droits humains, à la critique de la dépolitisation de la notion d'empowerment en la rendant soluble dans les idées qui concernent le capital humain, et à la relation d'aide, foncièrement inégalitaire, il ne me semble pas que ces éléments suffisent à "penser l'empowerment comme critique radicale des différentes formes de domination, une critique au cœur du politique et à l'intersection des paradigmes" (p. I26).

\section{«Le care au secours de l'empowerment», vraiment?}

Je dois cependant admettre que j'ai été estomaquée par la proposition d'un care qui permettrait de réduire certaines des apories de l'empowerment. L'argument le plus important que soutient Mestiri en faveur du care, c'est qu'il permet une "refonte de la traditionnelle dichotomie public/privé » (p. I27). Effectivement, si le care nous force à examiner sous un nouvel angle l'idéal d'autonomie prôné par les libéralismes classique, rawlsien et néolibéral, il ne s'ensuit pas pour autant qu'une notion égalitaire d'interdépendance puisse nécessairement y être associée.

Pour développer son idée de care, Mestiri s'appuie sur Joan Tronto, théoricienne reconnue dans ce domaine et sur laquelle Laugier, Papperman, Brugère ou Molinier se fondent pour tenter de développer à la fois une éthique et une politique du care. Elle tempère également son enthousiasme à propos des potentialités du care en faisant état de trois critiques qui montrent sa face obscure, critiques développées par Narayan. La première concerne le 
fait que la majeure partie des tâches associées au care sont effectuées par des femmes, très souvent d'ailleurs des femmes racisées. La deuxième a trait à une certaine marchandisation du care dans un contexte néolibéral. La troisième traite de la justification du colonialisme à l'aide d'un discours de care.

Mestiri insiste également, dans la deuxième partie du chapitre VI sur la nécessité de distinguer la solidarité de la charité. Le care doit donc être distingué du don puisqu'il repose sur la notion de réciprocité. S'il est plausible de soutenir que tous les êtres humains sont vulnérables pour mettre en évidence sa dimension de réciprocité, il n'en reste pas moins que dans les sociétés réelles, au Nord comme au Sud, le care bénéficie à des êtres humains parfaitement valides (des hommes blancs en santé) et vulnérabilise économiquement, politiquement et sexuellement les êtres humains qui effectuent majoritairement ce care (principalement des femmes, souvent racisées). Pour répondre à cette objection qu'elle n'ignore pas, Mestiri nous offre le raisonnement paradoxal suivant: une politisation du care qui fait de l'empowerment l'incarnation de la réciprocité que nous recherchons» (p. I36, souligné dans le texte), et elle poursuit en soutenant que cela "suppose de définir les modalités d'une émancipation mutuelle et véritable au-delà de la simple empathie» (p. I37). Mais l'on peut penser que, contrairement à ce qui est souhaité par Mestiri, à savoir une prise de conscience de la vulnérabilité de chaque personne, nous risquons fort d'être confronté.es plutôt à une confirmation des privilèges de ceux qui peuvent exiger du care sans contrepartie.

Mestiri avance quelques arguments intéressants concernant le traduisible et le traductible qui implique, contrairement au premier terme, une écoute attentive de l'autre. Elle en appelle également à repenser les liens entre morale et politique, entre morale et affects, et finalement entre sphère privée et sphère publique de l'existence humaine, mais faire jouer ces différenciations dans une perspective décoloniale ne signifie pas nécessairement pouvoir en finir avec les diverses dominations. Cela ne fait que mettre en évidence qu'il y a là un terrain d'affrontement social.

\section{Conclusion}

Avec cet ouvrage, Mestiri apporte une contribution importante au féminisme décolonial, même si, comme plusieurs autres, elle soulève plus de questions qu'elle n'apporte de réponses. Elle a au moins le mérite de poser des questions fondamentales pour l'avenir du féminisme sur le plan théorique aussi bien que pratique, puisqu'elle se situe sur le terrain de la philosophie sociale.

Cependant, les audaces décoloniales sont souvent contrecarrées par un enracinement théorique dans le libéralisme rawlsien qui permet difficilement de penser les rapports sociaux et les luttes d'émancipation. Par ailleurs, remplacer l'autonomie du sujet libéral par une interdépendance permettant la construction de solidarités est un projet essentiel pour penser un monde sans domination. Là encore, le libéralisme rawlsien est de peu d'utilité. 